\title{
A criterium for monomiality
}

\author{
Carolina Vallejo Rodríguez
}

ABstRact - Let $G$ be a solvable group. An odd degree rational valued character $\chi$ of $G$ is induced from a linear character of some subgroup of $G$. We extend this result to odd degree characters $\chi$ of $G$ that take values in certain cyclotomic extensions of $\mathrm{Q}$.

Mathematics Subject Classification (2010). 20C15.

KEYwords. Monomial characters, characters of finite groups.

\section{Introduction}

It follows from a well-known result of R. Gow [1] that an odd-degree rational-valued irreducible character of a solvable group is monomial. In this note, we slightly generalize Gow's result.

If $\chi$ is an irreducible complex character of a finite group $G$, let

$$
n(\chi)=\operatorname{gcd}\left(n \mid \mathrm{Q}(\chi) \subseteq \mathrm{Q}_{n}\right),
$$

where $Q(\chi)$ is the smallest field containing the values of $\chi$, and we denote by $Q_{n}$ the $n$-th cyclotomic field.

Theorem A. Let $G$ be a solvable group. Let $\chi \in \operatorname{Irr}(G)$. If $\chi(1)$ is odd and $\operatorname{gcd}(\chi(1), n(\chi))=1$, then there exist a subgroup $U \subseteq G$ and a linear character $\lambda$ of $U$ such that $\lambda^{G}=\chi$. Moreover, if $\mu$ is a linear character of some subgroup $W \subseteq G$ and $\mu^{G}=\chi$, then there exists $g \in G$ such that $W=U^{g}$ and $\mu=\lambda^{g}$.

(*) Indirizzo dell'A.: Departamento de Álgebra, Facultad de Ciencias Matemáticas C/ Dr. Moliner, 50 C.P. 46100 Burjassot, Spain.

E-mail: carolina.vallejo@uv.es

This research is partially supported by Proyecto MTM2010-15296 of the Spanish Government. 
Theorem $\mathrm{A}$ is not true if $\chi(1)$ is even or if $G$ is not solvable $\left(S L_{2}(3)\right.$ has a rational valued non-monomial character of degree 2 , and $A_{6}$ has a rational valued non-monomial character of degree 5).

I would like to thank M. Isaacs and G. Navarro for simplifications of earlier versions of the proof of this result.

\section{Proof of Theorem A}

We use the notation of [2]. First, we prove by induction on $|G|$ that $\chi$ is monomial.

STEP 1. We may assume $\chi$ is faithful and that there is no proper subgroup $H<G$ and $\psi \in \operatorname{Irr}(H)$ such that $\psi^{G}=\chi$ and $Q(\psi) \subseteq Q(\chi)$.

Let $K=\operatorname{ker} \chi$. If $K>1$, all the hypotheses hold in $G / K$ so by induction we are done. For the second part $\psi(1)$ divides $\chi(1)$, and $n(\psi)$ divides $n(\chi)$, so $\operatorname{gcd}(\psi(1), n(\psi))=1$, and the inductive hypothesis applies.

\section{STEP 2. $\mathbf{F}(G)=\prod_{p \nmid \chi(1)} \mathbf{O}_{p} G$.}

Let $p$ be a prime. Suppose that $p$ divides $\chi(1)$. In particular $p$ is odd, and $p$ does not divide $n(\chi)$. Let $M$ be a normal $p$-subgroup of $G$. Since $\mathrm{Q}(\chi) \subseteq \mathrm{Q}_{n}(\chi)$ we have that $\mathrm{Q}_{|M|} \cap \mathrm{Q}(\chi)=\mathrm{Q}$. Hence $\chi_{M}$ is rational-valued. If $\xi \in \operatorname{Irr}(M)$, then $\left[\chi_{M}, \xi\right]=\left[\chi_{M}, \bar{\xi}\right]$. Since $\chi(1)$ is odd, there exists a real irreducible constituent $\xi$ of $\chi_{M}$. Since $|M|$ is odd, we have that $\xi=1_{M}$, by Burnside's theorem. By Step 1, we know that $\chi$ is faithful and we conclude $M=1$.

STEP 3. $F=\mathbf{F}(G)$ is abelian.

Let $M$ be a normal $p$-subgroup of $G$, where $p$ does not divide $\chi(1)$. It then follows that the irreducible constituents of $\chi_{M}$ are linear. Let $\lambda \in \operatorname{Irr}(M)$ be under $\chi$. We have that $M^{\prime} \subseteq \operatorname{ker} \lambda^{g}=\operatorname{ker} \lambda^{g}$ for every $g \in G$. Then $M^{\prime} \subseteq \operatorname{core}_{G}(\operatorname{ker}(\lambda)) \subseteq \operatorname{ker}(\chi)=1$, so that $M$ is abelian. Hence $F$ is abelian by Step 2 .

SteP 4. Let $N \triangleleft G$ and let $\theta \in \operatorname{Irr}(N)$ be under $\chi$. Let $g \in G$. Then $\theta^{g}=\theta^{\sigma}$ for some $\sigma \in \operatorname{Gal}(\mathrm{Q}(\theta) / \mathrm{Q})$. Also $\theta$ is faithful.

Let $T=I_{G}(\theta)$ be the stabilizer of $\theta$ in $G$, and write $T^{*}$ for the semi-inertia subgroup of $\theta$, this is $T^{*}=\left\{g \in G \mid \theta^{g}=\theta^{\sigma}\right.$ for some $\left.\sigma \in \operatorname{Gal}(\mathrm{Q}(\theta) / \mathrm{Q})\right\}$. By part (b) of Lemma 2.2 of [3], if $\psi \in \operatorname{Irr}(T \mid \theta)$ is the Clifford correspondent 
for $\chi$, then $\eta=\psi^{T^{*}} \in \operatorname{Irr}\left(T^{*}\right)$ induces $\chi$ and $\mathrm{Q}(\eta)=\mathrm{Q}(\chi)$. By Step 1, we have that $T^{*}=G$. So that every $G$-conjugate of $\theta$ is actually a Galois conjugate. Thus $\operatorname{ker} \theta^{g}=\operatorname{ker} \theta$ for every $g \in G$. It follows that $\operatorname{ker}(\theta) \triangleleft G$ and $\operatorname{ker}(\theta)$ is contained in $\operatorname{ker}(\chi)$ by Clifford's theorem. So $\theta$ is faithful by Step 1.

STEP 5. If $\lambda \in \operatorname{Irr}(F)$ is under $\chi$, then $\lambda^{G}=\chi$.

Let $\lambda \in \operatorname{Irr}(F)$ be under $\chi$. If $y \in G$ is such that $\lambda^{y}=\lambda$, then we have $[x, y] \in \operatorname{ker}(\lambda)$ for every $x \in F$. By step $4, \lambda$ is faithful, so the element $y$ centralizes $F$. Since $F$ is self-centralizing, necessarily $y \in F$. We have proved $I_{G}(\lambda)=F$. This implies $\lambda^{G}$ is irreducible and thus $\lambda^{G}=\chi$. This finishes the proof that $\chi$ is monomial.

Now, we work by induction on $|G|$ to show that if $U$ and $V$ are subgroups of $G$ and $\lambda \in \operatorname{Irr}(U)$ and $\mu \in \operatorname{Irr}(V)$ are linear such that $\lambda^{G}=\chi=\mu^{G}$, then the pairs $(U, \lambda)$ and $(V, \mu)$ are $G$-conjugate. Since $K=\operatorname{ker}(\chi) \subseteq \operatorname{core}_{G}(\operatorname{ker}(\lambda)) \cap \operatorname{core}_{G}(\operatorname{ker}(\mu))$ we may assume that $\chi$ is faithful, for if $K>1$ then we can work in $G / K$. If $p$ is a prime not dividing $\chi(1)$, then $\mathbf{O}_{p}(G)$ is contained in both $U$ and $V$, because $|G: U|=$ $\chi(1)=|G: V|$. By Step 2 (which only required that $\chi$ is faithful), we have that

$$
\mathbf{F}(G)=\prod_{p \nmid \chi(1)} \mathbf{O}_{p}(G) \subseteq U \cap V .
$$

Now $\lambda_{F}$ and $\mu_{F}$ are both under $\chi$. So that $\mu_{F}=\left(\lambda_{F}\right)^{g}$ for some $g \in G$ by Clifford's theorem. We may assume that $\mu_{F}=\lambda_{F}=v$, by replacing the pair $(U, \lambda)$ by some $G$-conjugate. Thus $U$ and $V$ are contained in $T=I_{G}(v)$ and also in $T^{*}$, the semi-interia subgroup of $v$. Since $\lambda^{G}$ and $\mu^{G}$ are irreducible, also $\lambda^{T}$ and $\mu^{T}$ are irreducible. By uniqueness of the Clifford correspondent, we deduce that $\lambda^{T}=\mu^{T}$. In particular $\lambda^{T^{*}}=$ $\mu^{T^{*}}=\psi \in \operatorname{Irr}\left(T^{*} \mid v\right)$. We know that $\mathrm{Q}(\psi)=\mathbb{Q}(\chi)$, again using Lemma 2.2 of [3]. If $T^{*}<G$, then the result follows by induction. Hence, we may assume $T^{*}=G$. In particular, arguing as in the first part of the proof, we conclude that $v^{G}=\chi$. This implies that $U=F=V$ and the theorem is proven.

Recently we have given a related criterium for monomiality in which the odd degree hypothesis is replaced by certain oddness related to Sylow normalizers (see [4]). This result and our Theorem A seem to be independent. Under the hypothesis of Theorem A, it can also be proved that in fact $\chi$ is supermonomial, that is, that every character inducing $\chi$ is monomial. 


\section{REFERENCES}

[1] R. Gow, Real valued Characters of Solvable Groups, Bull. London Math. Soc., 7 (1975), p. 132.

[2] I. M. IsAACS, Character Theory of Finite Groups, AMS Chelsea Publishing, 2006.

[3] G. Navarro - J. Tent, Rationality and Sylow 2-subgroups, Proc. Edinburgh Math. Society. 53 (2010), pp. 787-798.

[4] G. Navarro - C. VAllejo, Certain monomial characters, Archiv der Mathematik. 99 (2012), pp. 407-411.

Manoscritto pervenuto in redazione il 27 Marzo 2013. 